\title{
Polarimetric Littrow Spectrograph - instrument calibration and first measurements ${ }^{\star}$
}

\author{
C. Beck ${ }^{1}$, W. Schmidt ${ }^{1}$, T. Kentischer ${ }^{1}$, and D. Elmore ${ }^{2}$ \\ 1 Kiepenheuer-Institut für Sonnenphysik, Schöneckstr. 6, 79104 Freiburg, Germany \\ e-mail: cbeck, wolfgang, tk@kis. uni-freiburg. de \\ 2 High Altitude Observatory, National Center for Atmospheric Research ${ }^{\star \star}$, 3450 Mitchell Lane, Boulder, Colorado 80301, \\ USA \\ e-mail: elmore@ucar.edu
}

Received 10 January 2005 / Accepted 5 April 2005

\begin{abstract}
We present first measurements and the calibration procedure for the Polarimetric Littrow Spectrograph (POLIS) operated at the Vacuum Tower Telescope on Tenerife, together with a brief summary of the technical characteristics of the instrument. In its present configuration, we achieve a polarimetric accuracy of about $3 \times 10^{-3}$ in the visible channel $(630 \mathrm{~nm})$ of the instrument. The accuracy is limited by cross talk among the different polarization states. The detection limit for polarized light is about $2 \times 10^{-3}$ for a $7 \mathrm{~s}$ exposure. Polarimetric measurements in the blue channel (Ca II $\mathrm{H}$ line, $396.7 \mathrm{~nm}$ ) are strongly limited by the low photon flux. At this wavelength we present Stokes- $V$ maps with a spatial resolution of about 0.5 arcs. The polarimetric quality of any spectropolarimeter is limited by the precision of the instrument calibration. We present a new method for self-calibration that reduces cross talk among the polarization components to $0.1 \%$. This improvement results from a measurement of the retardance of the calibration waveplate with an accuracy of $0.1^{\circ}$. We demonstrate the capability of the simultaneous use of POLIS and the Tenerife Infrared Polarimeter which is integrated in the main spectrograph of the Vacuum Tower Telescope.
\end{abstract}

Key words. Sun: magnetic fields - instrumentation: polarimeters

\section{Introduction}

The existence, spatial and temporal distribution of the magnetic field in the solar photosphere is often measured with magnetographs, originally developed around 1953 (Kiepenheuer 1953; Babcock 1953). For a proper understanding of the processes that lead to the variety of magnetic objects and features on the sun, it is necessary to measure the magnetic field vector, i.e. magnitude and orientation of the field, with high precision, using a vector-polarimeter. A number of these instruments are presently operated at several solar observatories, e.g., the Advanced Stokes Polarimeter (Skumanich et al. 1997) and the Diffraction-Limited Stokes Polarimeter (Sankarasubramanian et al. 2003) at the Dunn Solar Telescope of the National Solar Observatory, the ZIMPOL-instruments (Gandorfer \& Povel 1997; Gandorfer et al. 2004) that have been used at different telescopes, the Tenerife Infrared Polarimeter (TIP) (Martínez Pillet et al. 1999) at the German Vacuum Tower Telescope (VTT), the spectro-polarimetric mode of the THEMIS solar

\footnotetext{
* Appendices are only available in electronic form at http: //www. edpsciences.org

$\star \star$ The National Center for Atmospheric Research is sponsored by the National Science Foundation.
}

telescope (López Ariste et al. 2001; Mein 2002), and the SOLIS Spectro-Polarimeter (Keller \& SOLIS Team 2003). All these instruments measure the Stokes vector, i.e. the polarization state of the incident light in one or several spectral lines. They differ mainly in the technical realization of the polarization modulation.

The Polarimetric Littrow Spectrograph (Schmidt et al. 2003) is designed for strictly simultaneous measurements of the Stokes vector in the photosphere and the chromosphere. In addition, the instrument is set up such that it can be operated together with the TIP. In that configuration, the vertical structure of the solar atmosphere can be probed using four different magnetically sensitive lines, with heights of formation from the deep photosphere to the mid chromosphere.

This paper presents first scientific observations with POLIS and demonstrates the capability of the simultaneous use of POLIS and TIP. The calibration procedure of the spectropolarimeter is described. First, we present the main characteristics of the new instrument. Section 3 deals with the important topic of polarimetric calibration of the instrument. In that section we also describe a method to characterize the calibration unit itself. First observational results are presented in Sect. 4. 


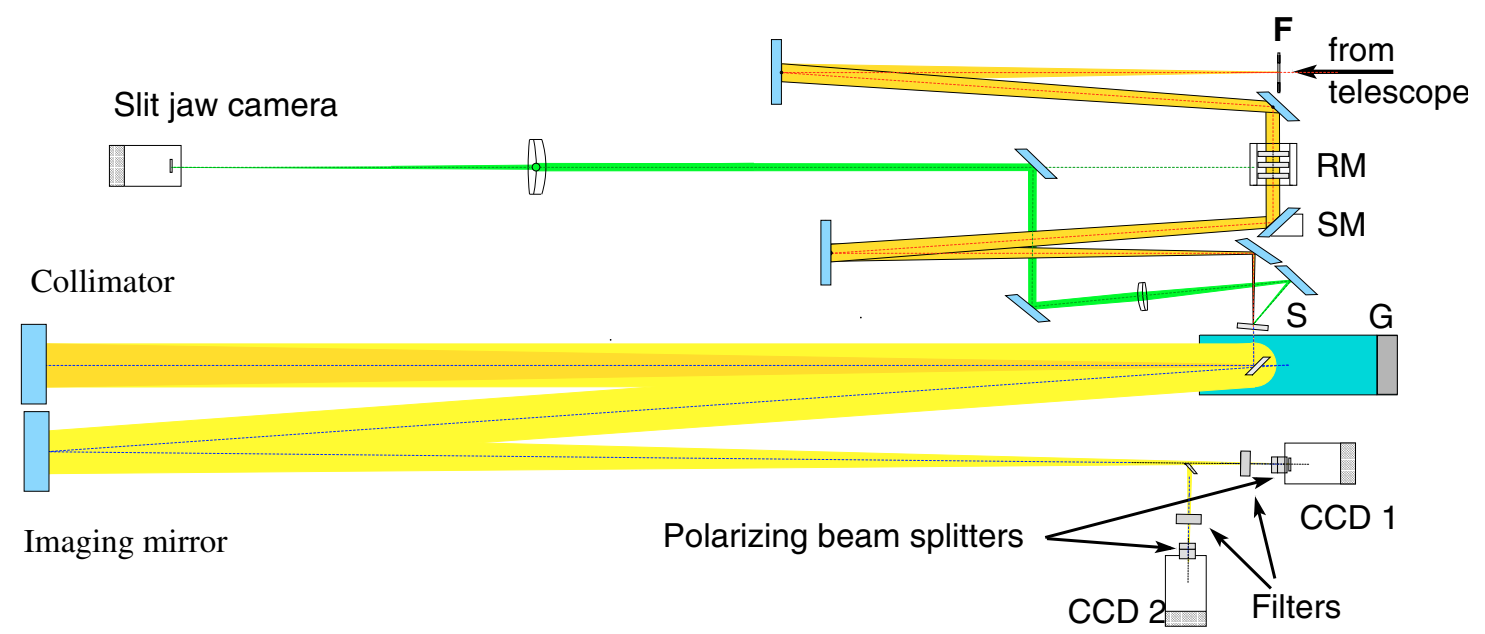

Fig. 1. Optical layout of POLIS. Light enters the instrument at top right. F denotes the telescope focal plane. A collimator mirror forms an image of the entrance pupil near the rotating modulator, RM, and the scan mirror, SM. An imaging mirror re-images the solar image at F to the entrance slit, S, of the spectrograph. Slit jaw images are recorded from the reflective slit jaws of the entrance slit. The collimator re-images the entrance pupil to the grating, G. The dispersed beams are then re-imaged to the focal plane detectors, CCD 1 and CCD 2. A small pickoff mirror close the focal plane reflects the blue beam to CCD 2. Diffraction orders are isolated with narrow-band filters placed in front of each detector. Polarizing beam splitters placed directly in front of the CCDs separate the orthogonally polarized beams.

The appendices provide important background material about the polarimeter response functions.

\section{Instrumental design and characteristics}

POLIS measures the Stokes vector $(I, Q, U, V)$ simultaneously in two wavelength regimes on two CCD cameras. One CCD records the polarization in two photospheric Fe I lines at $630.15 \mathrm{~nm}$ and $630.25 \mathrm{~nm}$ (in the following called red channel). The second camera records the line core and the blue wing of the $\mathrm{Ca}$ II $\mathrm{H}$ line at $396.8 \mathrm{~nm}$ (blue channel). The CCDs are back-side illuminated 14-bit PLUTO cameras from Pixel Vision, with a full detector size of $652 \times 488$ pixel, and a pixel size of $12 \mu \times 12 \mu$. The data can be binned internally over CCD columns and rows to increase the signal level. A slit jaw camera takes broad band images of the reflective front side of the slit. The wavelength of the slit jaw images is selected by means of an interference filter.

The red and the blue branches of the instrument are dual beam systems with polarizing beam splitters in front of each CCD camera. The optical layout of POLIS is displayed in Fig. 1. The instrument consists of three major parts:

1. The scan unit, with the modulator and the scan mirror.

2. The slit jaw camera.

3. The main disperser, with slit, grating, polarizing beam splitters, narrow-band filters for order selection and the CCDs for the two channels.

The optical layout of the main disperser resembles that of a Littrow spectrograph: the light beams are in a plane perpendicular to the plane of dispersion. This allows for very small reflection angles at the collimating and imaging mirrors.

POLIS uses a single rotating retarder as polarization modulator that modulates the polarization signals in both wavelength bands with high efficiency. The modulator is a zero-order waveplate, equipped with a wedge to eliminate spectral fringes.
A new (patented) design for the rotating waveplate has been developed (Schmidt \& Kentischer 2003), which completely avoids any beam wobble caused by the rotating wedge. The wedge is compensated by two glass wedges in front and behind the retarder. The compensation of the beam displacement caused by the wedged modulator is achieved by fine-tuning of the orientation of the additional wedges. The resulting accuracy of the angular displacement compensation is much higher than the manufacturing tolerances of the wedges themselves, resulting in a wobble-free image. In addition, the final configuration has no parallel glass surfaces and therefore eliminates problems associated with interference fringes.

Polarizing beam splitters in front of the cameras split the modulated light beam in two orthogonally polarized beams $I^{ \pm}$, imaged on a single CCD in each channel. Two types of beam splitters are available splitting the beams along the spatial or spectral axis. Since the CCD is rectangular, this allows to choose a larger spatial or spectral field of view (see Table B.1 in the Appendix).

The intensity of the individual beams $I^{ \pm}$as a function of the incoming Stokes vector, the modulator angular position, $\theta$, and the retardance of the modulator, $\delta$, is given by:

$$
\begin{aligned}
I^{ \pm}(\theta, \delta)= & \frac{r^{ \pm}}{2}\left\{I \pm Q \cdot\left(\cos ^{2} 2 \theta+\sin ^{2} 2 \theta \cdot \cos \delta\right)\right. \\
& \pm U \cdot \sin 2 \theta \cdot \cos 2 \theta \cdot(1-\cos \delta) \mp V \cdot \sin 2 \theta \cdot \sin \delta\},
\end{aligned}
$$

where $r^{ \pm}$is the transmission of the respective beam. The ratio $r^{+} / r^{-}$is about 0.92 , mainly due to the different path length in the polarizing beamsplitters (Schmidt et al. 2001; Beck 2002). Equation (1) is an idealized equation which is valid under the assumption that the optics between modulator and beamsplitter do not change the polarization state. The dual beam design decreases the amount of seeing induced cross-talk and increases the detection efficiency (Lites et al. 1993). 
In order to derive the values of $I, Q, U$, and $V$, the intensity signal is demodulated in real-time after 8 images, taken at the modulator positions $k \times 22.5^{\circ}(k=0 \ldots 7)$, using a suitable summation scheme (Lites et al. 1993). The demodulation is expressed by an $8 \times 4$ matrix, applied to the 8 intensity images. The demodulation can be reversed again by the calculation of the inverse matrix by a singular value decomposition. In this paper, we do not use this option, but it would allow to perform additional corrections, e.g., dark current subtraction, on the single images, if necessary.

The total integration time can be varied by changing the modulator rotation frequency, $v_{\mathrm{m}}$, or by accumulating the demodulated Stokes vectors, $\mathrm{n}_{\mathrm{acc}}$ :

$t_{\text {exp }}=\frac{1}{2 \cdot v_{\mathrm{m}}} \cdot n_{\mathrm{acc}}$.

A single image has an integration time of $1 / 16 \cdot v_{\mathrm{m}}^{-1}$. The rotation frequency of the modulator can be varied from 0.1 to $3 \mathrm{~Hz}$, resulting in integration times between 625 and $20 \mathrm{~ms}$ for a single image. The disk-write time, $t_{\mathrm{w}}$, for one complete Stokes measurement, is in the order of $1 \mathrm{~s}$.

The scan unit is located in front of the entrance slit and allows to take raster images with a range of about \pm 100 arcs around the center position of the scan mirror. The smallest step width is 0.1 arcs.

Table B.1 lists the spectral and spatial properties of the two CCD cameras for the two possible orientation of the polarizing beam splitters. The observable spectral lines in the wavelength intervals are listed in Table B.2.

For the calibration of the instrument, an Instrument Calibration Unit (ICU) has been installed near the prime focus of the telescope. The ICU consists of a linear polarizer and a zero order quartz retarder. The optical elements of the ICU are mounted on a motorized stage and are moved in the beam together. The retarder and the polarizer can be rotated independently around the optical axis. The angular position accuracy is better than $0.1^{\circ}$.

\section{Calibration of polarimetric data}

In addition to the usual flatfield procedure (Sect. 3.1), polarimetric data has to be corrected for the influence of the telescope and the instrument itself on the measured polarization state. The relation between measured Stokes vector, $\boldsymbol{S}_{\text {out }}$, and the true solar polarization state before entering the telescope, $S_{\text {true }}$, can be expressed by

$S_{\text {out }}=X \cdot \mathcal{T} \cdot S_{\text {true }}$,

where $X$ accounts for all changes due to optics behind the ICU, and $\mathcal{T}$ includes the changes due to the telescope.

The derivation of $\boldsymbol{X}$ is described in Sect. 3.2 while the limitations in its determination are discussed separately in Sect. 4 because they restrict the final accuracy of the polarization measurement.

\subsection{Gain table correction}

In order to correct for any inhomogeneities of the raw images that may be caused by the detector itself, by vignetting

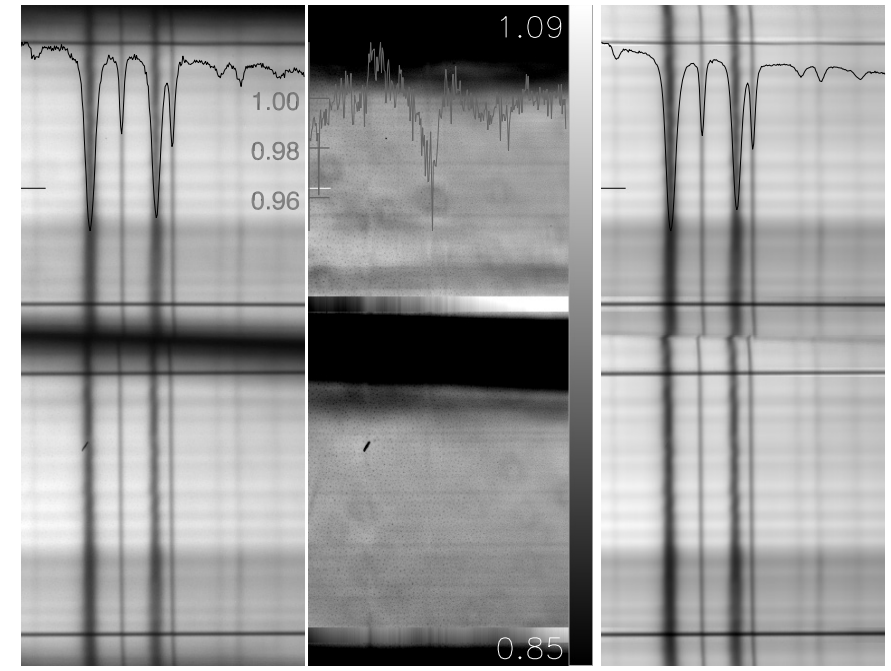

Fig. 2. Example of the gain table correction for the red channel, using data from August 2003. Image size $288 \times 652$ pixel. Left: uncorrected image, $I^{+}$and $I^{-}$are separated spatially. Middle: Gain table. Right: Gain-corrected image. Profiles of each image from the marked row (short bar at left) are overplotted.

of the beam or other imperfections, a gain table has to be constructed. To this end, about 50 flat-field images are taken near disk center, with a random telescope pointing to provide a homogeneous illumination free of solar surface structure. The observed wavelength is not changed, therefore, the raw flat-field data contains the same spectral lines as the science data (Fig. 2, left panel).

The next step toward the construction of the gain table is the removal of the spectral line information from the flatfield images. The compact design with a short focal length of the spectrograph leads to a curvature of the spectral lines along the slit (variation of the line core position of $\sim 3$ pixel). Additionally, intensity gradients along the slit, and at the borders of the beams are present. This requires the application of a rather complicated procedure for the removal of the spectral information. The spectra are divided in a number of stripes along the slit. Within these stripes, the line curvature is removed, and the mean line profile is derived by averaging along the slit. The mean line profiles from the stripes are normalized to each other to remove the intensity variation along the slit. The gain table for each stripe is then obtained by dividing the spectrum by the averaged line profile.

The two beams are treated separately, and then normalized in intensity to each other as last step of the gain correction. Figure 2 displays an example of the gain table used for data of August 2003, and a spectrum before and after the gain correction. The gaintable takes into account only pixel-to-pixel variations of the intensity response. Intensity gradients in wavelength direction (cf. the profiles in Fig. 2) are not corrected for because they were found to depend on the polarization state.

\subsection{Derivation of the polarimeter response function}

The instrument calibration unit is used to generate different known polarization states to retrieve the response function of 
the instrument, $\boldsymbol{X}$. High precision of the calibration requires good knowledge of the polarizing properties of the elements of the ICU, and of the orientation of the polarizer transmission axis.

The orientation of the axes has been measured in the laboratory using standard techniques with an accuracy of $\pm 0.1^{\circ}$ for the polarizer, and $\pm 1^{\circ}$ for the retarder. The retardance value has been measured with an indirect method, described later in Sect. 4 , as $(61.5 \pm 0.1)^{\circ}$ at $630 \mathrm{~nm}$. The measurement of the retardance at $400 \mathrm{~nm}$ was not successful in the laboratory, due to the low light level of the lamp used. We therefore measured the retardance at several wavelengths and extrapolated a value of $114.5^{\circ}$ for $400 \mathrm{~nm}$.

From the measured angular orientation of the elements, and modeling the ICU elements as ideal polarizer and non-ideal retarder (cf. Eq. (A.1)), we compute the polarization state of the beam that passes the ICU. A comparison with the actually measured Stokes vector yields the polarimeter response function.

Although we use solar light from nonmagnetic regions, the light incident on the ICU is already partially polarized by oblique reflections in the telescope. These effects are taken into account by the application of the telescope Mueller matrix, $\mathcal{T}$, to the solar illumination, like in Eq. (3).

Thus, the general equation between the solar input, $\left(\begin{array}{llll}I_{0} & 0 & 0 & 0\end{array}\right)^{T}$, and measured output, $S^{\text {out }}$, during the calibration is given by:

$$
\begin{aligned}
\boldsymbol{S}^{\text {out }} & =\boldsymbol{X} \boldsymbol{M}_{\mathrm{r}}\left(\theta_{r}\right) \boldsymbol{M}_{\mathrm{p}}\left(\theta_{p} \cdot \mathcal{T} \cdot\left(\begin{array}{llll}
I_{0} & 0 & 0 & 0
\end{array}\right)^{T}\right. \\
& =I_{0} \cdot \boldsymbol{X} \boldsymbol{M}_{\mathrm{r}}\left(\theta_{\mathrm{r}}\right) \boldsymbol{M}_{\mathrm{p}}\left(\theta_{\mathrm{p}}\right) \cdot\left(\begin{array}{llll}
1 & \mathcal{T}_{2,1} & \mathcal{T}_{3,1} & \left.\mathcal{T}_{4,1}\right)^{T},
\end{array}\right.
\end{aligned}
$$

where $\theta_{\mathrm{p}}$ and $\theta_{\mathrm{r}}$ are defined as the angles between terrestrial E-W and the transmission axis of the polarizer and the fast axis of the retarder. $\boldsymbol{M}_{i}\left(\theta_{i}\right)$ are the Mueller matrices of the rotated optical elements. The total transmission of the telescope, $\mathcal{T}_{1,1}$, has been set to 1 . If the linear polarizer is kept fixed, e.g., at $\theta_{\mathrm{p}} \equiv 0^{\circ}$, Eq. (4) simplifies to:

$\boldsymbol{S}^{\text {out }}=I_{0} \cdot \frac{1}{2}\left(1+\mathcal{T}_{2,1}\right) \cdot \boldsymbol{X} \boldsymbol{M}_{\mathrm{r}}\left(\theta_{\mathrm{r}}\right) \cdot\left(\begin{array}{llll}1 & 1 & 0 & 0\end{array}\right)^{T}$.

The variation of $\mathcal{T}_{2,1}$ during the time needed for a calibration measurement is small. Keeping the polarizer fixed completely decouples the polarimeter calibration from the telescope polarization because a fixed polarization state with almost constant light level is created behind the polarizer.

A standard calibration data set consists of one half rotation of the retarder in $5^{\circ}$-steps. The calibration input for $\theta_{\mathrm{p}} \equiv 0^{\circ}$ is thus given by:

$S_{\mathrm{c}}^{\text {in }}=M_{\mathrm{r}}\left(0^{\circ}, 5^{\circ}, \ldots, 180^{\circ}\right) \cdot\left(\begin{array}{llll}1 & 1 & 0 & 0\end{array}\right)^{T}$.

To derive the observed Stokes spectra, $S_{\mathrm{c}}^{\text {out }}(\lambda)$, the individual images of the two beams are corrected for dark current and gain inhomogeneities, then balanced in intensity with the ratio $r^{+} / r^{-}$derived from the normal flat-field data, and finally merged (Beck 2002). The spectra are then spatially and spectrally averaged. Intensity trends during the calibration due to the change of $\mathcal{T}_{2,1}$ are removed by a normalization with a linear regression to the 37 Stokes $I$ values.

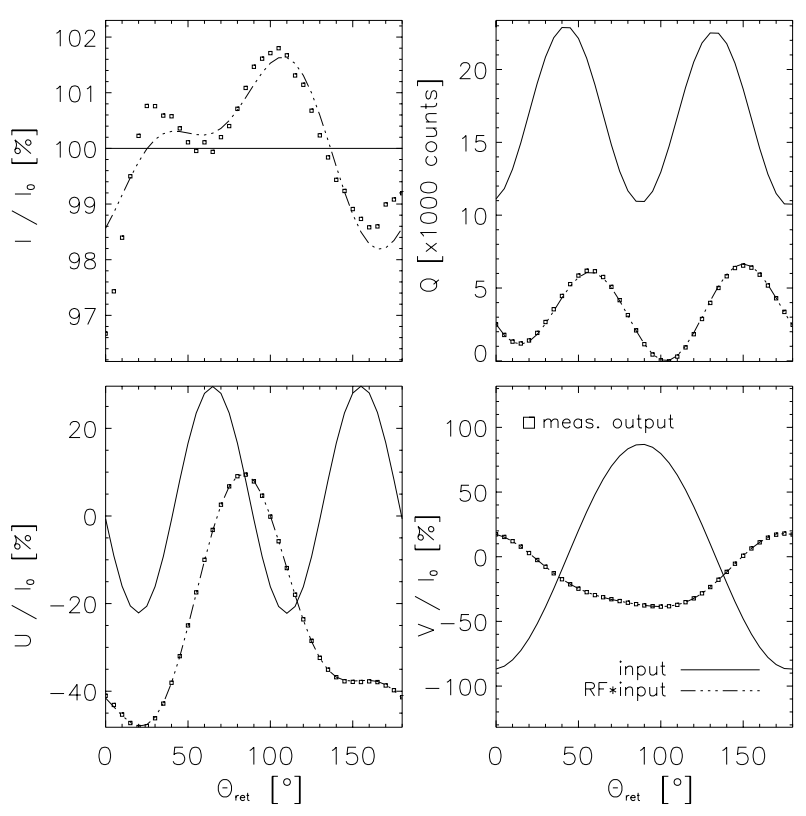

Fig. 3. Stokes vector $(I, Q, U, V)$ of a calibration measurement in the red channel. Solid: calibration input vector, calculated from the retarder and polarizer position angles. Squares: measured Stokes vector. Dash-dotted: Computed output vector $\boldsymbol{S}_{\mathrm{c}}^{\text {out }}$ (Eq. (7)). Note the good agreement with the measured curve. Stokes $Q$ is given in digital counts instead of the normalized intensity for a comparison with the corresponding number in the blue channel (cf. Fig. A.1).

The polarimeter response function is derived from the measurements after arranging the Stokes vectors into $37 \times 4$ matrices by a solution of the linear problem

$S_{\mathrm{c}}^{\text {out }}=S_{\mathrm{c}}^{\text {in }} \cdot X^{T}$

The solution method by matrix inversion and the error estimate of the procedure can be found in Appendix A. A comparison between generated input, measured Stokes vector, and the transformation of input to output by the response function is displayed in Fig. 3. The largest deviations between measured and predicted values are found in Stokes $I$ which is less constrained than the polarization components due to its smaller variation during the calibration.

In addition to the 16 elements of $\boldsymbol{X}$, there are two free parameters:

- linear dichroism, $b$, of the retarder (cf. Skumanich et al. 1997, p. 363, or Eq. (A.1)),

- an offset angle, $\theta_{\text {off }}$, between ICU polarizer and retarder to account for misalignment.

The free parameters are computed by minimizing the $\chi^{2}$ value

$\chi^{2}\left(b, \theta_{\text {off }}\right)=\sum_{\mathrm{i}=0.3}\left(\boldsymbol{S}_{\mathrm{c}}^{\text {out }}-\boldsymbol{S}_{\mathrm{c}}^{\mathrm{in}}\left(b, \theta_{\mathrm{off}}\right) \boldsymbol{X}^{\mathrm{T}}\right)_{\mathrm{i}}^{2}$,

with respect to $b$ and $\theta_{\text {off }}$ by a gradient method. A change of $b$ or $\theta_{\text {off }}$ only affects $S_{\mathrm{c}}^{\text {in }}$. The maxtrix inversion itself is "instantaneous", i.e. it at once yields the best-fit solution if $\boldsymbol{S}_{\mathrm{c}}^{\text {in }}$ and $\boldsymbol{S}_{\mathrm{c}}^{\text {out }}$ are given. 
The response function and the free parameters are measured for typically 4-8 intervals along the slit, in order to account for a possible variation of the instrument properties in the spatial direction. A typical result for the red channel is:

$\boldsymbol{X}=\left(\begin{array}{rrrr}1.000 & 0.0504 & 0.0063 & -0.0258 \\ -0.0078 & 0.0993 & -0.3218 & 0.3891 \\ -0.0208 & -0.4448 & -0.2443 & -0.0943 \\ -0.0150 & -0.1833 & 0.2281 & 0.2914\end{array}\right)$

It shows significant cross talk values between Stokes $V$ and $Q\left(\mathrm{X}_{2,4}\right)$. The value of $\theta_{\text {off }}$ was $-1.5^{\circ}$, and $b$ was -0.00382 . This corresponds to a minor deviation of the waveplate from the ideal case.

The total efficiency, $e_{i}$, for measuring a Stokes parameter is derived from the corresponding column of the response function by

$e_{i}=\sqrt{\sum_{j=1 . .4} X_{j, i}^{2}}$,

which yields for the matrix in Eq. (9) a value of $\left(e_{Q}, e_{U}, e_{V}\right)=$ (0.494, 0.464, 0.496).

For the blue channel, the simultaneously taken data have a very low light level that does not allow to derive a reliable response function. Results for the calibration of the blue channel of POLIS from another data set with longer integration time are shown in Appendix A.3.

\subsection{Instrumental polarization of the VTT}

Since the ICU is mounted near the prime focus of the telescope, it does not calibrate the polarimetric properties of the the telescope itself. Therefore, a model of the polarization properties of the VTT Tenerife has been developed. This model delivers the Mueller matrix, $\mathcal{T}$, of the telescope, for a given observation time. It includes all optical elements of the telescope up to the ICU position. The telescope model and its verification will be described in a separate paper (Beck et al. 2005, subm.).

\subsection{Calibration of solar observations}

The calibration of solar observations includes the following steps:

1. Dark current and gain table correction in each beam, balancing of intensity of the two beams,

2. removal of intensity gradients in wavelength dimension by applying a 3rd order polynomial to continuum windows,

3. spatial and spectral alignment of the two beams,

4. merging of aligned beams,

5. application of the inverse matrices $\mathcal{T}^{-1} \cdot \boldsymbol{X}^{-1}$ to the measured Stokes vector.

The alignment is done with sub-pixel precision using cubic interpolation of the data. For the spatial alignment the hairlines are used which are located at the entrance focal plane of POLIS.

\section{Calibration accuracy and sensitivity}

\subsection{Polarimetric accuracy}

The polarimetric accuracy of the calibration depends strongly on the parameters which enter the calculation of the response function through the constructed Stokes input vectors, $S_{\mathrm{c}}^{\mathrm{in}}$.

The absolute error of the matrix inversion (cf. Appendix A) is below $8 \times 10^{-4}$ for the matrix derived above. This contribution can be neglected, since the magnitude of the fit error does only reflect the value of $\chi^{2}$ from Eq. (8) which due to the number of "free" parameters always is very small.

The main contribution to the calibration error - and hence to the quality of the calibrated data - stems from the poorly known retardance of the calibration waveplate.

We had measured the retardance directly, with the retarder placed between crossed polarizers, and found a value of $63.5^{\circ} \pm 1^{\circ}$ which was not accurate enough for our needs. The polarimeter calibration procedure itself offers a second method with a much higher sensitivity and accuracy. Since the reference system for the Stokes vectors is fixed in space, the result of the response function must not depend on the actual orientation of the polarizer during the calibration measurement. Therefore we carried out several calibration measurements with the polarizer angle increased in $10^{\circ}$-steps. The evaluation of the data using different retardance values showed that the value of the matrix elements varied with the polarizer orientation, except for a retardance value of $61.5^{\circ}$. The effect is most pronounced in the first column of $\boldsymbol{X}$. Figure 4 displays the effect of retardance changes on the matrix element $X_{3,1}, I \rightarrow U$. Other matrix elements (e.g. $X_{2,1}$ and $X_{4,1}$ ) show a similar behavior, consistent with a retardance of $61.5^{\circ}$.

The indirect method described above allows to determine the retardance of a waveplate with an accuracy of $0.1^{\circ}$. This is much better than typical manufacturing tolerances that are in the order of $1^{\circ}$. The accuracy of $0.1^{\circ}$ leads to a polarimetric accuracy of $1.4 \times 10^{-3}$ (cf. the differences of the curves for $61.5^{\circ}$ and $61.4^{\circ}$ in Fig. 4). From measurements in different seasons it was found that the actual retardance value of the ICU retarder depends slightly on the light level in the telescope, i.e. due to the increased illumination in summer - and thus a higher temperature of the retarder due to absorption - the retardance is then smaller by about $0.2^{\circ}-0.4^{\circ}$. Taking this temperature effect into account the effective error limit should be around $3-5 \times 10^{-3}$. Note that this is the error in the determination of the relative cross-talk, not of the absolute accuracy of the measurements.

\subsection{Polarimetric sensitivity}

The polarimetric sensitivity is defined as the smallest detectable signal of solar origin. With an integration time of $0.8 \mathrm{~s}$ and 8 accumulations of the demodulated Stokes vector (=total exposure of $6.4 \mathrm{~s}$ ) the smallest "clear" signal detected in a single resolution element was $2 \times 10^{-3}$ at a noise rms level of $9 \times 10^{-4}$. For this exposure time the sensitivity limit is of the same order as the error in the response function which deals with the cross talk among the components of the Stokes vector. 


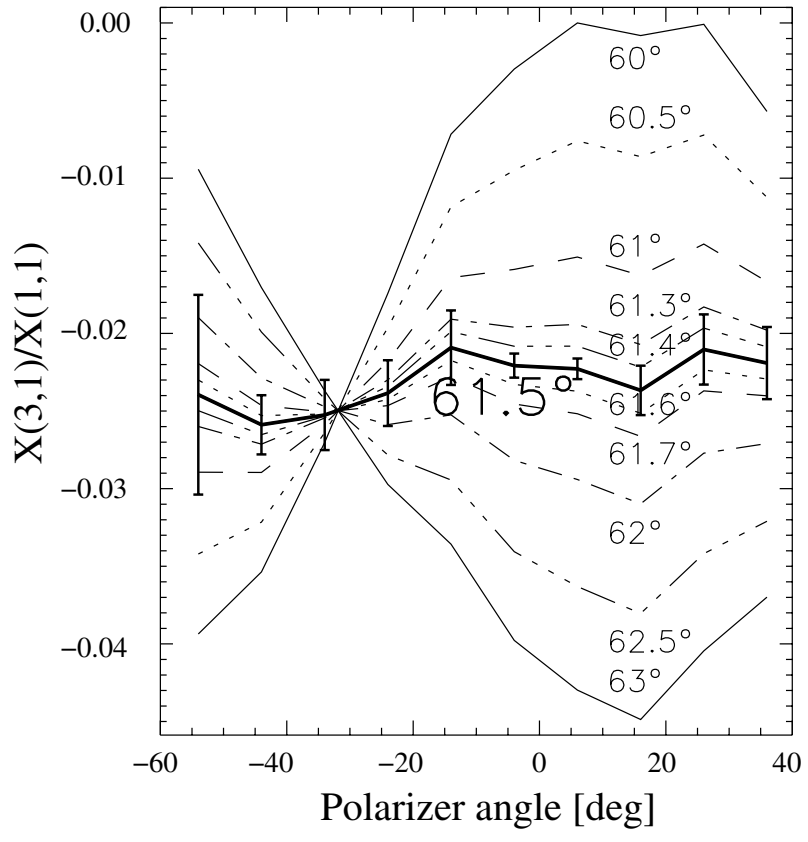

Fig. 4. Derivation of the retardance of the calibration waveplate. The matrix element $X_{3,1}$ of the instrument response function (Stokes $I \rightarrow$ $U$ ) is plotted as a function of polarizer position angle for different values of the retardance. From the shape of the curves we find a "best" retardance of $61.5^{\circ}$. The fit error is indicated for the $61.5^{\circ}$ curve.

\section{Observations}

Two observing campaigns were carried out in 2003. The first one was used to obtain calibration data for telescope and polarimeter, and to test the possibility of simultaneous observations with the Tenerife Infrared Polarimeter (TIP). In the second campaign we explored the capabilities and limitations of polarimetric measurements in the $\mathrm{Ca}$ II $\mathrm{H}$ line. Additionally, some simultaneous maps with TIP were taken.

\subsection{Stokes profiles in the red channel}

Figure 5 shows one of the observed regions, NOAA 0425 . The spot was scanned with a step width of 0.35 arcs, an integration time per step of $3.3 \mathrm{~s}$ and a slit width of 0.48 arcs. The light level in POLIS in this observation was reduced by a factor of 2 due to the 50-50-beamsplitter used to simultaneously feed TIP. Figure 6 displays Stokes profiles from single spatial pixels in the umbra and the penumbra of this spot, and from the surrounding granulation. The spectral sampling is shown in the Stokes $V$ profile of the penumbra. In the umbra the Zeeman components of the most magnetic sensitive line at $630.25 \mathrm{~nm}$ are resolved in the intensity profile. The noise rms in the continuum polarization is about $3 \times 10^{-3} \cdot I_{\mathrm{c}}$.

\subsection{Stokes $\mathrm{V}$ observations in $\mathrm{Ca} \| \mathrm{H}$}

The light level in the core of the $\mathrm{Ca}$ II $\mathrm{H}$ line, together with decreased reflectivities of the reflective surfaces are limiting the performance of the blue channel of POLIS. Several measures had been taken to improve the transmission of the VTT such

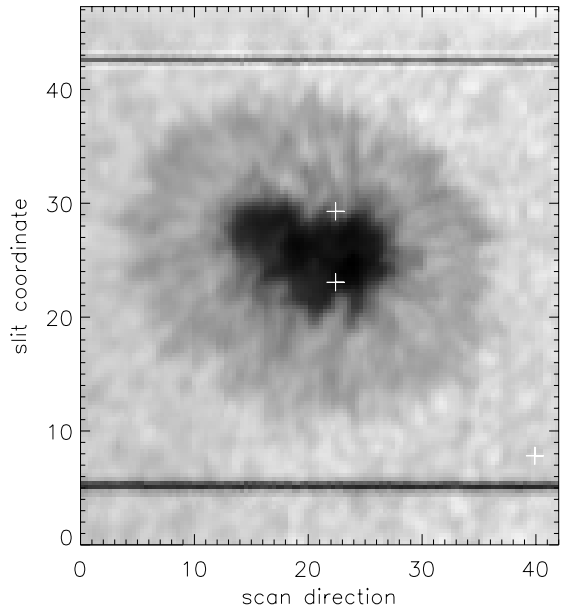

Fig. 5. Intensity map of sunspot NOAA 0425 , observed on 9.8.2003, between 7:40 and 7:52 UT. Tick marks correspond to 1 arcs. Scan direction left to right, step width 0.35 arcs, 120 steps. The intensity gradient in scan direction is caused by the increase of intensity in the early morning. White crosses indicate the spatial positions of the profiles shown in Fig. 6.

that polarization measurements in the $\mathrm{Ca}$ II $\mathrm{H}$ line core became possible.

Figure 7 displays the result of a short scan of two small pores. Data were taken with an exposure time of $26.7 \mathrm{~s}$ and a slit width of 0.48 arcs. The 50-50-beamsplitter was exchanged with a flat mirror. The chromospheric calcium line is in emission for most of the surroundings of the pores, with two separate emission peaks outside and a single emission peak inside the pores. The line core of calcium shows a sign reversal in Stokes $V$ in the center of the pores.

Figure 8 displays an example of such co-spatial line profiles at $630 \mathrm{~nm}$ and at $396.7 \mathrm{~nm}$, taken from an individual resolution element of $0.48 \times 0.292 / 0.145 \mathrm{arcs}^{2}$ for the blue/red channel. The noise rms of the red channel has decreased to about $5 \times 10^{-4} \cdot I_{\mathrm{c}}$ through the longer exposure time. The emission core of the calcium line at $396.88 \mathrm{~nm}$ shows a pronounced Stokes- $V$ signal with the opposite sign compared to the photospheric absorption lines in the wing of the calcium line or in the red channel. The light level was insufficient to detect any clear Stokes $Q$ or $U$ signal in the calcium line core. It will require further technical improvements of the telescope and the instrument to increase the light level by a factor of, say, four, to allow for "true" Stokes vector measurements in the blue channel of POLIS.

Figure 9 displays simultaneous data from TIP and POLIS of a scan over a part of the active region NOAA 0484, taken on 20.10.2003 at 10:11 UT. The exposure time was again $26.7 \mathrm{~s}$ per scan step. This spot group was connected with X-ray emission and flare activity throughout its disk passage. The profiles of the blue channel in Fig. 10 show very strong emission in the calcium line in part of the area.

\section{Summary}

We have presented the calibration method and first observations with POLIS, part of them taken simultaneously with the 

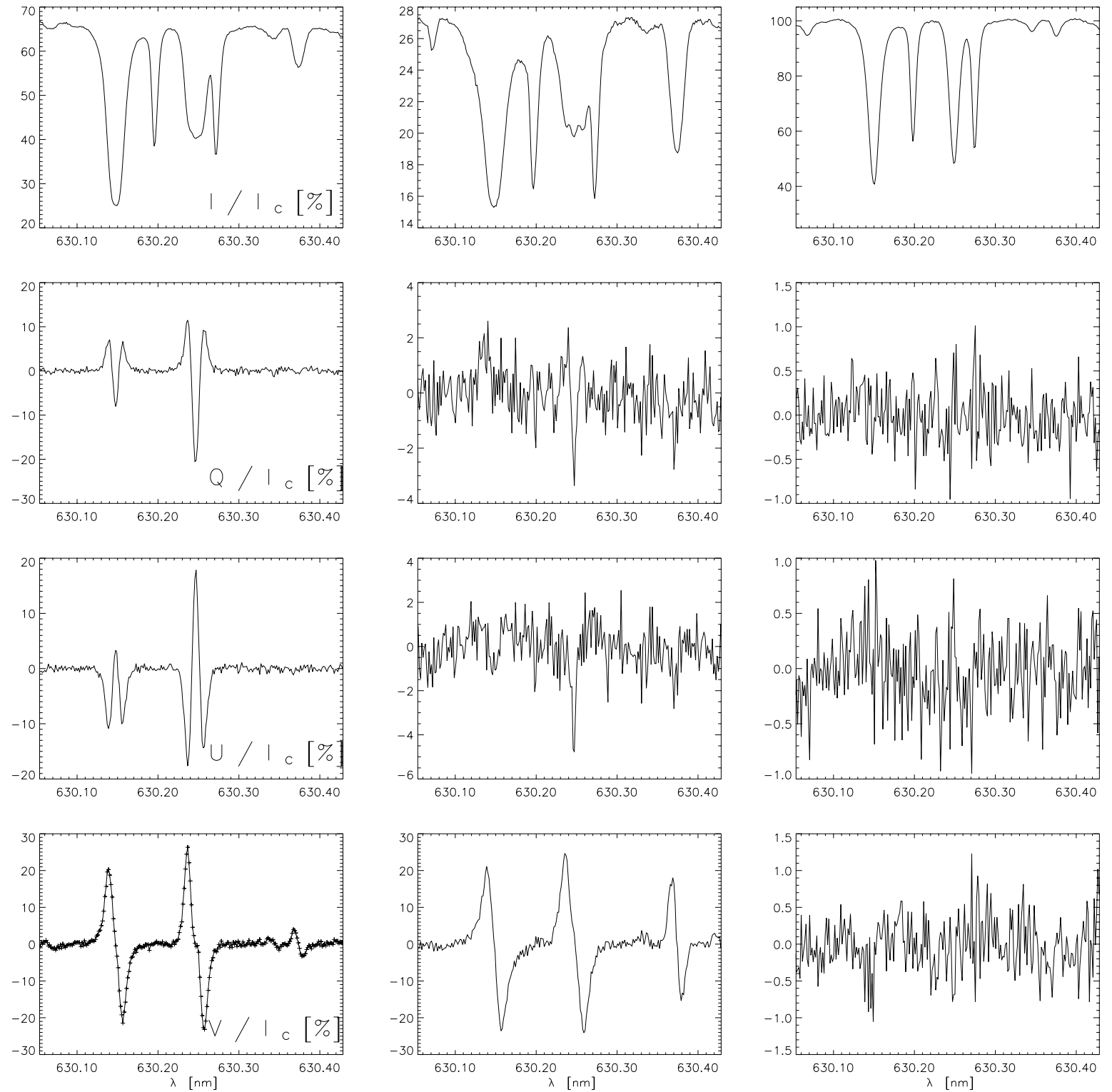

Fig. 6. Stokes profiles measured in the red channel, top to bottom: $I, Q, U, V$ each normalized to continuum intensity, $I_{\mathrm{c}}$, of the quiet Sun profile. Left column: sunspot penumbra, middle column: umbra, right column: quiet Sun. The profiles were taken at the points marked with crosses in Fig. 5.

TIP. We demonstrate that the instrument - in its present configuration - is capable to perform full Stokes vector polarimetry with an accuracy of $3 \times 10^{-3} \cdot I$ at $630 \mathrm{~nm}$, with a spatial sampling of 0.145 arcs per pixel in the red channel.

We have developed a powerful method to calibrate the retardance of our calibration waveplate with an accuracy of $0.1^{\circ}$. The value of this retardance affects directly the polarimetric accuracy, since it determines the cross-talk correction.

With the present light level, we are able to measure Stokes $V$ in Ca II $\mathrm{H}$ profiles, with a spatial resolution of about 0.5 arcs and an exposure time of $\sim 27 \mathrm{~s}$.

This is quite unsatisfactory, and additional efforts are planned to further increase the light level. If photons were the only criterion, one could more or less easily switch to one of the infrared calcium lines around $850 \mathrm{~nm}$. One of the important criterions to choose the $\mathrm{Ca}$ II $\mathrm{H}$ line in the first place was the availability of magnetically sensitive lines in the wings of the line. The simultaneous measurement of chromospheric and photospheric lines at the same wavelength guarantees that the information is indeed taken at exactly the same loaction on the sun. Otherwise, one has to measure or compute the differential refraction effects with great care.

An IDL-based software package has been developed for all steps of the photometric and polarimetric calibration. This software is available to all users of POLIS. A software code for simultaneous observations with the TIP has been implemented at the control computers.

First observations with this instrument have demonstrated that it is a very powerful tool for the investigation of the solar magnetic field at different heights in the solar atmosphere, especially in combination with the TIP to cover the deep photospheric layers. A new beamsplitter is available in the meantime 

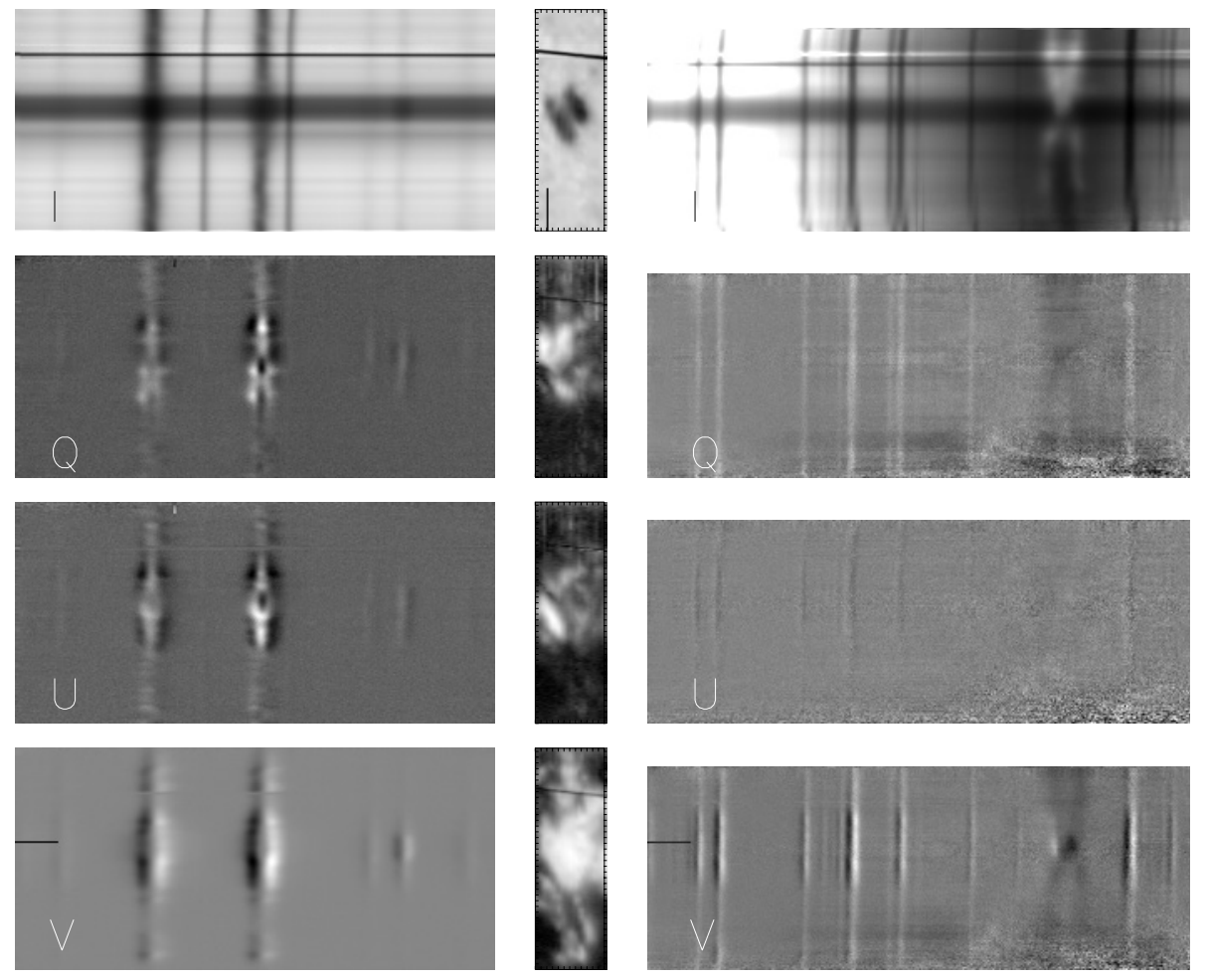

Fig. 7. Scan of a small pore, observed on 15 Oct. 2003. Top to bottom: Stokes components $I, Q, U$ and V. Left column: spectra of Fe I 630.15 and Fe I $630.25 \mathrm{~nm}$, single scan step. Middle column: wavelength integrated maps of Stokes $I, Q . U$ and $V$ around $630.2 \mathrm{~nm}$. The vertical black bar in $I$ indicates the scan position of the spectra shown. Tick marks correspond to 1 arcs. Right column: spectra of the Ca II H line. The horizontal bar in the Stokes $V$ spectrum marks the position of the profiles displayed in Fig. 8. Only the co-spatial field-of-view of blue and red channel is shown. The red channel has been rescaled to have the same spatial sampling of 0.29 arcsec per pixel.
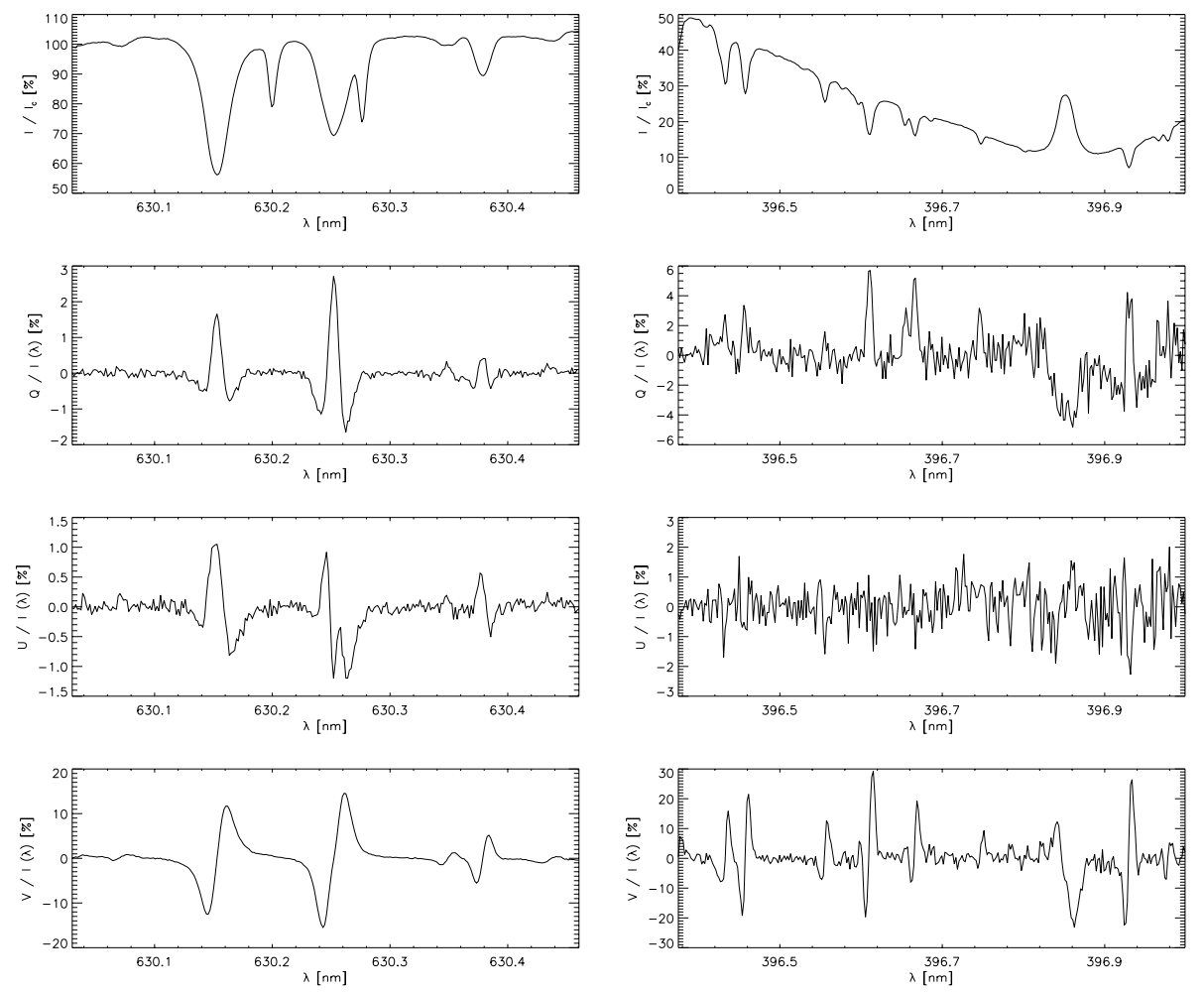

Fig. 8. Co-spatial profiles in red (left column) and blue (right column) channel of POLIS, top to bottom: Stokes $I, Q, U$ and $V$. Data were taken on 15 Oct. 2003 at 15:30 UT, scan of a small pore. Note the opposite sign of the Stokes $V$ signal in the calcium line core, compared to that of the photospheric lines in the wing of the calcium line or in the red channel. The apparent reversal is due to the emission profile in the calcium core that changes the sign of the circular polarization. 

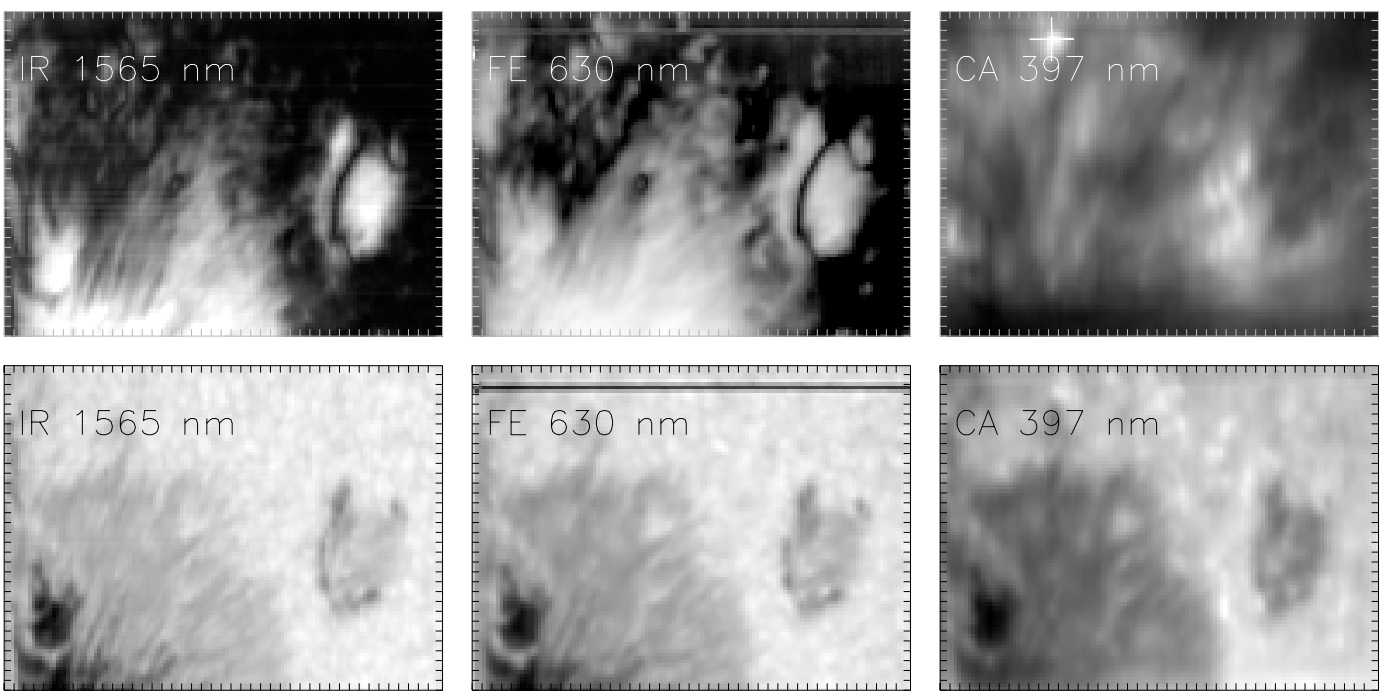

Fig. 9. Simultaneous data from TIP and POLIS, showing part of active region NOAA 0484, observed on 20 Oct. 2003 at 10:11 UT at the VTT Tenerife. Tick marks are 1 arcs. Bottom row, left to right: intensity maps in Fe I $1565 \mathrm{~nm}$ (integrated over the line), continuum at $630.3 \mathrm{~nm}$, and in the blue wing of the Ca II H $396.5 \mathrm{~nm}$ line. Top row, left to right: total circular polarization $\int|V|(\lambda) \mathrm{d} \lambda$ for TIP and the red channel of POLIS, intensity map of the $\mathrm{H} 2 \mathrm{v}$ emission peak at $396.83 \mathrm{~nm}$ of the blue channel. The white cross marks the spatial position of the line profile shown in Fig. 10.

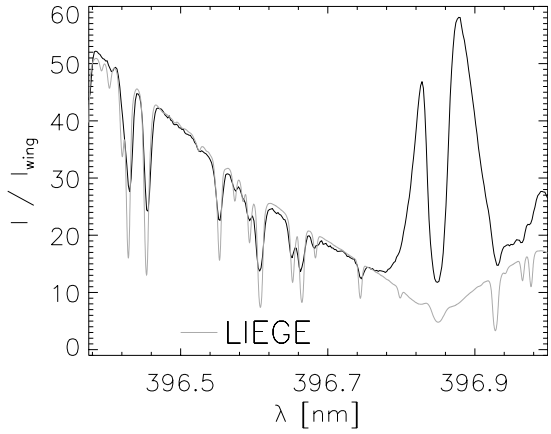

Fig. 10. Intensity spectrum of the blue channel corresponding to the strongest emission observed in the map of Fig. 9. Thin grey line: Liège atlas profile (Delbouille et al. 1973).

which splits the light around $800 \mathrm{~nm}$, i.e. all wavelengths below are reflected to POLIS while the rest is transmitted to TIP. This will increase the light level in both instruments and allow for shorter exposure times with a signal-to-noise ratio still better than in the present setup.

Acknowledgements. We thank Manolo Collados, Thomas Keller and Kim Streander for their support during the observations, and Luis Bellot Rubio, Rolf Schlichenmaier and the referee for useful comments. This work was supported by the Deutsche Forschungsgemeinschaft under grant Schl 514/2-1.

\section{References}

Babcock, H. W. 1953, ApJ, 118, 387

Beck, C. 2002, Diploma thesis, Univ. Freiburg: The calibration of the vectorpolarimeter POLIS,

http://www.kis.uni-freiburg.de/cbeck_diplom.pdf
Beck, C., Schlichenmaier, R., Collados, M., Bellot Rubio, L. R., \& Kentischer, T. 2005, A\&A, in preparation

Delbouille, L., Roland, G., \& Neven, L. 1973, Atlas photométrique DU spectre solaire de [lambda] 3000 a [lambda] 10000 (Liège: Université de Liège, Institut d'Astrophysique)

Gandorfer, A. M., \& Povel, H. P. 1997, A\&A, 328, 381

Gandorfer, A. M., Steiner, H. P. P. P., Aebersold, F., et al. 2004, A\&A, 422, 703

Keller, C. U., \& SOLIS Team. 2003, AAS/Solar Physics Division Meeting, 34, 0

Kiepenheuer, K. O. 1953, ApJ, 117, 447

López Ariste, A., Socas-Navarro, H., \& Molodij, G. 2001, ApJ, 552, 871

Lites, B. W., Elmore, D. F., Seagraves, P., \& Skumanich, A. P. 1993, ApJ, 418, 928

Martínez Pillet, V., Collados, M., Sánchez Almeida, J., et al. 1999, in High Resolution Solar Physics: Theory, Observations, and Techniques, ASP Conf. Ser., 183, 264

Mein, P. 2002, A\&A, 381, 271

Nave, G., Johansson, S., Learner, R. C. M., Thorne, A. P., \& Brault, J. W. 1994, ApJS, 94, 221

Pierce, A. K., \& Breckenridge, J. B. 1974, The Kitt Peak table of photographic solar spectrum wavelengths (Kitt Peak National Observatory Contribution, Tucson: Kitt Peak National Observatory, 1973-1974)

Sankarasubramanian, K., Elmore, D. F., Lites, B. W., et al. 2003, in Polarimetry in Astronomy, ed. S. Fineschi, Proc. SPIE, 4843, 414

Schmidt, W., \& Kentischer, T. 2003, in Dt. Patent 10236999

Schmidt, W., Kentischer, T. J., Bruls, J., \& Lites, B. W. 2001, in Advanced Solar Polarimetry - Theory, Observation, and Instrumentation, ASP Conf. Ser., 236, 49

Schmidt, W., Beck, C., Kentischer, T., Elmore, D., \& Lites, B. 2003, Astron. Nachr., 324, 300

Skumanich, A., Lites, B. W., Martínez Pillet, V., \& Seagraves, P. 1997, ApJS, 110, 357

Solanki, S. K., Pantellini, F. G. E., \& Stenflo, J. O. 1987, Sol. Phys., 107, 57 


\section{Online Material}




\section{Appendix A: Derivation of the response function}

\section{A.1. ICU retarder}

The retarder of the ICU is modelled by

$$
\boldsymbol{M}_{\mathrm{r}}=\left(\begin{array}{cccc}
1 & b / 2 & 0 & 0 \\
b / 2 & 1 & 0 & 0 \\
0 & 0 & \alpha \cdot \cos \delta_{\mathrm{r}} & -\alpha \cdot \sin \delta_{\mathrm{r}} \\
0 & 0 & \alpha \cdot \sin \delta_{\mathrm{r}} & \alpha \cdot \cos \delta_{\mathrm{r}}
\end{array}\right)
$$

with the retardance of the waveplate, $\delta_{\mathrm{r}}$, the linear dichroism, $b$, and $\alpha=\sqrt{1-b^{2} / 4}$. $\alpha$ is close to one even for large $b$.

\section{A.2. Matrix inversion and error estimate}

The polarimeter RF can be derived from a calibration data set by arranging the input, $S_{\mathrm{c}}^{\text {in }}(=\boldsymbol{A})$, and output, $\boldsymbol{S}_{\mathrm{c}}^{\text {out }}(=\boldsymbol{y})$, into $37 \times 4$-matrices. The resulting linear problem

$S_{\mathrm{c}}^{\text {out }}=S_{\mathrm{c}}^{\mathrm{in}} \cdot \boldsymbol{X}^{\mathrm{T}} \equiv \boldsymbol{y}=\boldsymbol{A} \cdot \boldsymbol{X}^{\mathrm{T}}$.

can be solved using $\boldsymbol{A}^{\mathrm{T}} \cdot \boldsymbol{y}=\boldsymbol{A}^{\mathrm{T}} \cdot \boldsymbol{A} \cdot \boldsymbol{X}^{\mathrm{T}}=\boldsymbol{D} \cdot \boldsymbol{X}^{\mathrm{T}}$ with:

$\boldsymbol{X}^{\mathrm{T}}=\boldsymbol{D}^{-1} \cdot \boldsymbol{A}^{\mathrm{T}} \cdot \boldsymbol{y}$.

$\boldsymbol{X}$ is a $4 \times 4$-matrix of the polarimeter response to polarized input, the individual columns give the response to a pure input state $I, Q, U$, or $V$. The error is estimated from $\boldsymbol{B}=\boldsymbol{D}^{-1} \cdot \boldsymbol{A}^{\mathrm{T}}$ in the following way:

$$
\begin{aligned}
\sigma_{X_{i}}^{2} & =\sum_{j} B_{i j}^{2} \sigma_{y_{j}}^{2}=\bar{\sigma}^{2} \sum_{j} B_{i j}^{2}, \text { where } \\
\bar{\sigma}^{2} & =\frac{1}{N} \cdot \sum_{\mathrm{cal}}\left(\boldsymbol{y}-\boldsymbol{A} \cdot \boldsymbol{X}^{\mathrm{T}}\right)^{2} .
\end{aligned}
$$

The errors of the single measurements, $\sigma_{y_{i}}$, are approximated by the total deviation of the fit, $\bar{\sigma}$. As the same $B_{i j}(j=$ $1, .,, 37)$ are used for the calculation of $\mathrm{X}_{i k}^{\mathrm{T}}(k=0,1,2,3)$, the procedure results in identical errors for each column of the polarimeter response function $\boldsymbol{X}$. The method is courtesy of M. Collados.

\section{A.3. Polarimeter response for the blue channel}

For calibration of the calcium channel usually a separate data set is taken with longer exposure time. A data set from 27.09.2004 with a rotation frequency of the modulator of $v_{\mathrm{m}}=$ $0.3 \mathrm{~Hz}$ and 16 accumulations of the Stokes vector (total integration time $27 \mathrm{~s}$ ) yielded the following polarimeter response function:

$\boldsymbol{X}=\left(\begin{array}{cccc}1.000 & 0.139 & -0.084 & 0.006 \\ 0.002 & 0.007 & 0.009 & -0.028 \\ 0.018 & -0.021 & -0.079 & -0.007 \\ 0.002 & -0.059 & 0.018 & 0.002\end{array}\right)$.

Dichroism, $b=-0.27$, offset angle $\theta_{\text {off }}=-7.64^{\circ}(!)$, fit error $\sim 0.3 \%$. The measured offset angle is greater than a single step of the retarder during the measurement. The efficencies are $\left(e_{Q}, e_{U}, e_{V}\right)=(0.153,0.117,0.030)$.
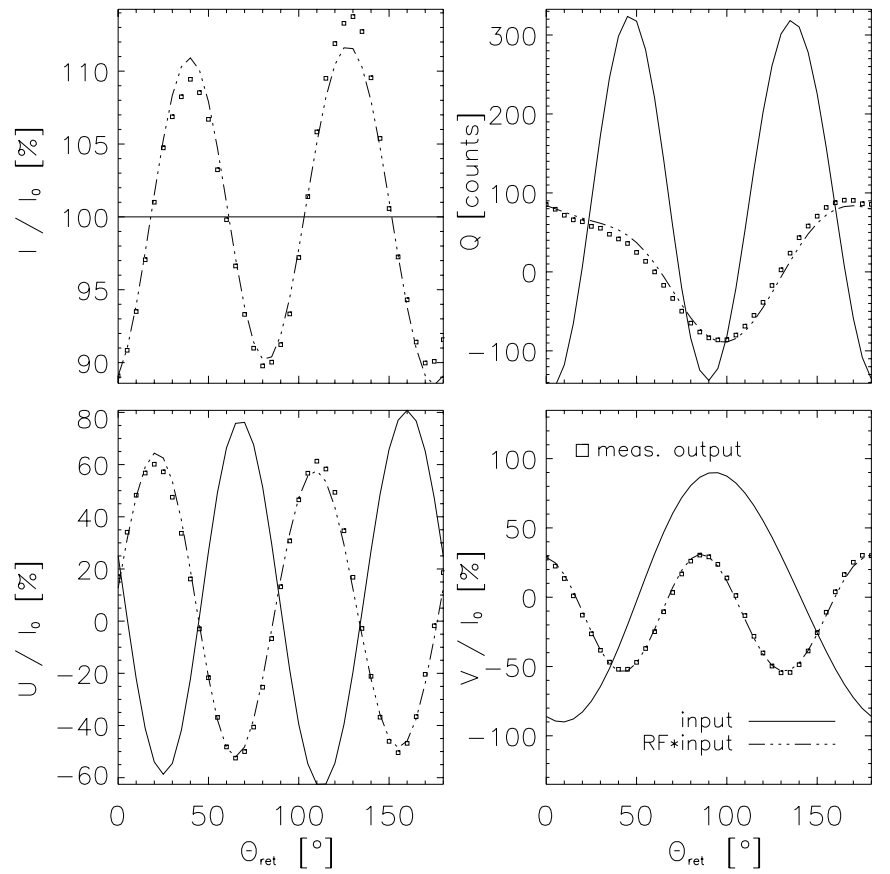

Fig. A.1. Same as Fig. 3 for the blue channel, Stokes vector $(I, Q, U, V)$ during the calibration. Solid: calibration input vector. Squares: measured Stokes vector. Dash-dotted: Computed output vector $\mathbf{S}_{\mathrm{c}}^{\text {out }}$ (cf. Eq. (7)). Stokes $Q$ is given in digital counts instead of the normalized intensity. Note that the observed polarization in $Q U V$ ( $\square$ ) has been multiplied with a factor of 10 for better visibility.

The corresponding curve of the Stokes vector during the calibration is displayed in Fig. A.1. The observed polarization level in $Q U V$ had to be increased by a factor of 10 to make the shape of the curve visible at all. The intensity is about 380 counts, while the maximum of, e.g., Stokes $Q$, does not exceed 9 counts. The smoothness of the curves results from the fact that each value of $Q U V$ for each calibration step is an average over 150 (in wavelength) $\times 40$ (along slit) $=6000$ pixels .

The concern about the correct retardance value mentioned in Sect. 4 of course also applies for the blue channel. Due to the lack of suitable data we had to use the extrapolated value of $114.5^{\circ}$ retardance without the possibility to confirm it by another method.

\section{Appendix B: Technical characteristics}

Table B.1 contains the spectral and spatial dimensions of the two POLIS channels, Table B.2 the observable spectral lines with some of their characteristics. 
Table B.1. Spectral and spatial properties of POLIS data for the two types of polarizing beam splitters in the red channel.

\begin{tabular}{|c|c|c|c|}
\hline Camera & Red & Blue & Unit \\
\hline Dispersion & 1.50 & 1.92 & $\mathrm{pm} / \mathrm{pixel}$ \\
\hline Spatial sampling & 0.145 & 0.290 & arcs/pixel \\
\hline Detector full size & $488 \times 652$ & $652 \times 488$ & pixel \\
\hline \multicolumn{4}{|c|}{ spectral division } \\
\hline Wavelength & $630.1-630.35$ & $396.4-397$ & $\mathrm{~nm}$ \\
\hline Final image size & $160 \times 652$ & $326 \times 122$ & pixel \\
\hline Spectral $\times$ spatial & $0.24 \times 96$ & $0.6 \times 36$ & $\mathrm{~nm} \times \operatorname{arcs}$ \\
\hline \multicolumn{4}{|c|}{ spatial division } \\
\hline Wavelength & $630.1-630.5$ & - & $\mathrm{nm}$ \\
\hline Final image size & $288 \times 326$ & - & pixel \\
\hline Spectral $\times$ spatial & $0.4 \times 48$ & - & $\mathrm{nm} \times \operatorname{arcs}$ \\
\hline
\end{tabular}

Table B.2. Observable spectral lines in red and blue channel. ${ }^{a}$ Wavelengths, Pierce \& Breckenridge (1974). ${ }^{b}$ Effective Landé factor, Solanki et al. (1987). ${ }^{c}$ Height of line core formation above $\tau_{500 \mathrm{~nm}}=1$. ${ }^{d}$ all values in this section from J. Bruls (personal note). ${ }^{e}$ Values from H. Schleicher (personal note). ${ }^{f}$ Nave et al. (1994).

\begin{tabular}{|c|c|c|c|c|}
\hline Element & $\begin{array}{c}\lambda \\
{[\mathrm{nm}]}\end{array}$ & $\begin{array}{c}\text { Transition } \\
{ }^{2 S+1} L_{J}\end{array}$ & $g_{j}$ & $\begin{array}{c}\Delta h^{c} \\
{[\mathrm{~km}]}\end{array}$ \\
\hline \multicolumn{5}{|c|}{ Solar absorption lines ${ }^{d}, \mathrm{CCD} 2$} \\
\hline Ca II H & 396.849 & ${ }^{2} \mathrm{~S}_{0.5}-{ }^{2} \mathrm{P}_{0.5}$ & 1.33 & $1480-2130$ \\
\hline $\mathrm{Fe} \mathrm{I}$ & 396.452 & ${ }^{3} \mathrm{P}_{1}-{ }^{5} \mathrm{P}_{2}$ & 2.00 & $110-320$ \\
\hline $\mathrm{Fe} \mathrm{I}^{f}$ & 396.551 & ${ }^{5} \mathrm{D}_{3}-{ }^{5} \mathrm{D}_{4}$ & & - \\
\hline $\mathrm{Fe} I$ & 396.607 & ${ }^{3} \mathrm{~F}_{2}-{ }^{3} \mathrm{D}_{3}$ & 2.00 & $350-520$ \\
\hline $\mathrm{Fe} \mathrm{I}^{f}$ & 396.665 & ${ }^{5} \mathrm{D}_{0}-{ }^{5} \mathrm{~F}_{1}$ & & - \\
\hline $\mathrm{Fe} \mathrm{I}^{f}$ & 396.681 & ${ }^{3} \mathrm{D}_{2}-{ }^{5} \mathrm{~F}_{1}$ & & - \\
\hline Fe I & 396.745 & ${ }^{3} \mathrm{H}_{4}-{ }^{1} \mathrm{G}_{3}$ & 0.75 & $270-450$ \\
\hline $\mathrm{Fe} I$ & 396.792 & ${ }^{5} \mathrm{D}_{3}-{ }^{3} \mathrm{G}_{4}$ & 1.00 & $280-510$ \\
\hline $\mathrm{Fe} I$ & 396.918 & ${ }^{3} \mathrm{~F}_{4}-{ }^{3} \mathrm{~F}_{3}$ & 1.50 & $510-630$ \\
\hline $\mathrm{Fe} \mathrm{I}^{f}$ & 396.963 & ${ }^{3} \mathrm{D}_{3}-{ }^{3} \mathrm{H}_{4}$ & & - \\
\hline \multicolumn{5}{|c|}{ Solar absorption lines, CCD 1} \\
\hline $\mathrm{FeI}$ & $630.151^{a}$ & ${ }^{5} \mathrm{P}_{2}-{ }^{5} \mathrm{D}_{2}$ & $1.67^{b}$ & $300-500^{e}$ \\
\hline $\mathrm{FeI}$ & $630.250^{a}$ & ${ }^{5} \mathrm{P}_{1}-{ }^{5} \mathrm{D}_{0}$ & 2.50 & $200-460^{e}$ \\
\hline $\mathrm{Fe} I$ & 630.346 & ${ }^{5} \mathrm{G}_{6}-{ }^{5} \mathrm{G}_{5}$ & & - \\
\hline Ti I & 630.375 & ${ }^{3} \mathrm{~F}_{3}-{ }^{3} \mathrm{G}_{3}$ & & \\
\hline \multicolumn{5}{|c|}{ Telluric absorption lines, CCD 1} \\
\hline $\mathrm{O}_{2}$ & $630.200^{a}$ & - & - & earth atm. \\
\hline $\mathrm{O}_{2}$ & $630.276^{a}$ & - & - & earth atm. \\
\hline
\end{tabular}

